\title{
Medium- and Long-Chain Dicarboxylic Aciduria in Patients with Zellweger Syndrome and Neonatal Adrenoleukodystrophy
}

\author{
FRANCIS ROCCHICCIOLI, PATRICK AUBOURG, AND PIERRE F. BOUGNÊRES \\ Département de Biochimie (INSERM U 75), Faculté de Médecine Necker-Enfants Malades; Service de \\ Neuropédiatrie et Service d'Endocrinologie (INSERM U 188), Hôpital Saint-Vincent-de-Paul, \\ Paris, Cédex, France
}

\begin{abstract}
This study reports that patients with neonatal adrenoleukodystrophy and Zellweger syndrome excrete a very peculiar pattern of organic acids. Dicarboxylic acids with an even number of carbon atoms (adipic, suberic, sebacic, 2- and 3-hydroxy-sebacic, hexadecanedioic), as well as with an odd number of carbon atoms (pimelic, azelaic, un-, tri-, and pentadecanedioic) were found in excess in the urines of six patients with neonatal adrenoleukodystrophy and one with Zellweger syndrome. The accumulation of dicarboxylic acids, reflecting an impairment of their $\beta$-oxidation in mitochondria and/or peroxisomes, thus appears as an additional useful marker of these peroxisomal diseases. (Pediatr Res 20: 62-66, 1986)
\end{abstract}

Abbreviations

BOHB, $\beta$-hydroxy-butyrate

BSTFA, N, O-bis-(trimethylsilyl)-trifluoroacetamide

DA, dicarboxylic acids

FA, fatty acids

FFA, free fatty acids

GC/MS, gas chromatography/mass spectrometry

LCFA, long chain fatty acids

MTBSTFA, N-methyl-N-(t-butyldimethylsilyl)-trifluoroacetamide

NALD, neonatal adrenoleukodystrophy

TBDMCS, t-butyldimethylchlorosilane

TMCS, trimethylchlorosilane

TMS, trimethylsilyl

VLCFA, very long-chain fatty acids

ZS, Zellweger syndrome

In rat liver, $\beta$-oxidation of both VLCFA (1-3) and DA (4) occurs mostly in peroxisomes, only a minor fraction of these substrates being oxidized in mitochondria $(3,4)$.

The accumulation of VLCFA in brain, adrenal glands, fibroblasts, and plasma of patients with ZS and NALD (5-8) have been attributed to a blockade of the peroxisomal oxidation of these fatty acids (3).

We report here that odd- and even-numbered DA are excreted in excess in the urine of patients with ZS or NALD, which suggests that the peroxisomal and/or mitochondrial $\beta$-oxidation of DA is also impaired.

Received December 18, 1984; accepted August 21, 1985

Correspondence and reprints to Dr. Francis Rocchiccioli, Département de Biochimie, Faculté de Médecine Necker-Enfants Malades, 156, rue de Vaugirard, 75730 Paris Cédex 15, France.

\section{CASE REPORTS}

Table 1 summarizes the clinical and biological data in the seven patients. More detailed clinical and histopathological data have been reported elsewhere $(9,10)$. None of the patients had attack of drowsiness, vomiting, or episodes of hypoglycemia and acidosis during the course of the disease. The days before the study, all patients were eating a normal controlled diet providing a daily caloric intake of $100-120 \mathrm{kcal} / \mathrm{kg}$, with $2.5 \mathrm{~g} / \mathrm{kg}$ protein and $2 \mathrm{~g} / \mathrm{kg}$ lipid. Estimated dietary intake of phytol was less than $1 \mathrm{mg} / \mathrm{kg} /$ day. Patients 1,2 , and 5 received Phenobarbital and patients 3 and 4, Carbamazepine.

\section{MATERIALS AND METHODS}

Reagents. Malonic, glutaric, 2-methylglutaric, phenylsuccinic, adipic, pimelic, suberic, azelaic, sebacic, undecanedioic, dodecanedioic, tetradecanedioic and hexadecanedioic acids, ethyl acetate and pyridine were obtained from Fluka (Buchs, Switzerland), tridecanedioic and n-butylmalonic acids from Aldrich (Beerse, Belgium), BSTFA with $1 \%$ TMCS from Pierce (Rockford, IL), and MTBSTFA with $1 \%$ TBDMCS from Chrompack (Middleburg, The Netherlands).

Methods. The concentration of carnitine in plasma and urine was measured using a slight modification of the method of McGarry (11). Plasma VLCFA were measured using a previously reported GC/MS technique (5). FFA were analyzed as follows: $20 \mu \mathrm{l}$ plasma samples, obtained after overnight fasting, were added to $1 \mathrm{ml}$ distilled water, then acidified with hydrochloric acid ( $12 \mathrm{~mol} / \mathrm{liter})$. Nonadecanoic acid ( $50 \mu \mathrm{mol} / \mathrm{liter})$ was added as internal standard, and FFA were extracted in ethyl acetate, evaporated to dryness under $\mathrm{N} 2$ and derivatized with MTBSTFA/pyridine (1:1). C16:0, C16:1, C18:0, C18:1, and C18:2 FA concentrations were quantified by selected ion monitoring of their [M-57] ions. BOHB was measured from $50 \mu \mathrm{l}$ plasma samples by ethyl acetate extraction, esterification by BSTFA/pyridine (1:1) and selected ion monitoring of the [M15] ion using malonic acid as internal standard.

\section{ANALYSIS AND QUANTITATION OF URINARY ORGANIC ACIDS}

Urine samples were collected after overnight fasting, immediately frozen and kept at $-80^{\circ} \mathrm{C}$ until analysis. Creatinine was determined on a Astra 8 apparatus (Beckman, Brea, CA).

The extraction of organic acids was performed with slight modifications of a previously described technique (12): 2-methylglutaric, n-butylmalonic, and phenylsuccinic acids were added as internal standards to a volume of urine containing 1.5 to 4 $\mu \mathrm{mol}$ of creatinine, extracted in ethyl acetate, evaporated to 
Table 1. Clinical and biochemical findings

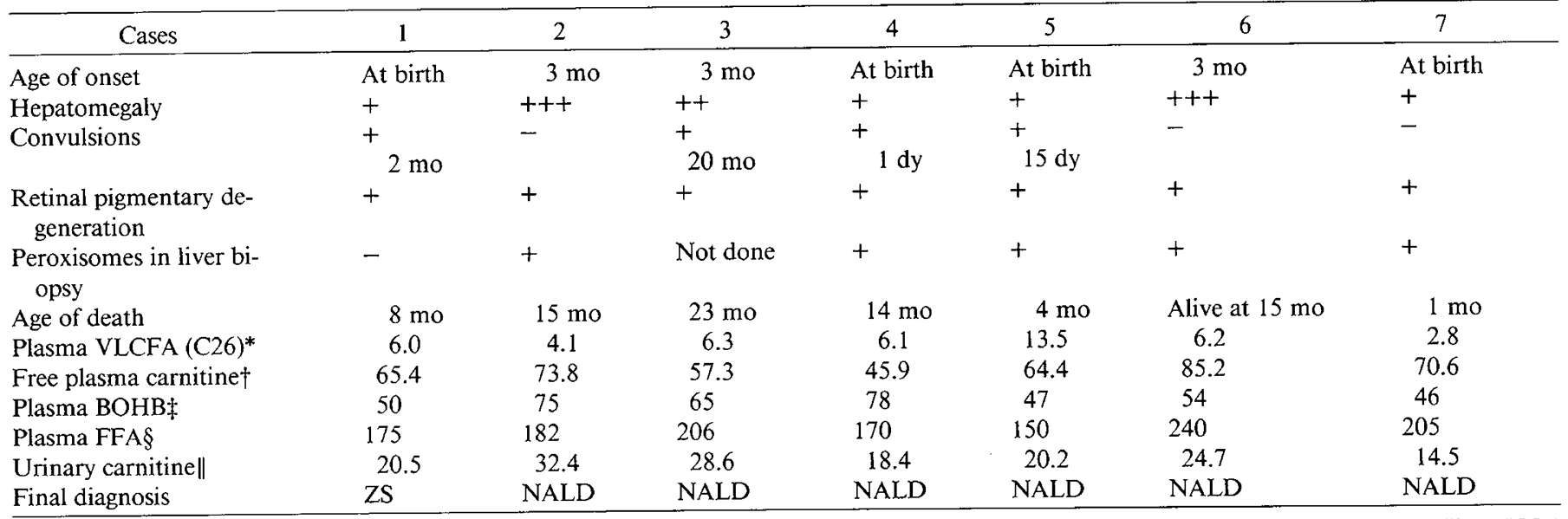

Normal values: * $0.35 \pm 0.19 \mu \mathrm{mol} /$ liter (Reference 9); $† 67.7 \pm 9.3 \mu \mathrm{mol} /$ liter (Reference 11 ); $\$ 61 \pm 23 \mu \mathrm{mol} /$ liter; $\S 204 \pm 47 \mu \mathrm{mol} /$ liter; $\| 25.1$ $\pm 6.1 \mu \mathrm{mol} / 24 \mathrm{~h}($ Reference 11$)$.

dryness and dissolved in $100 \mu \mathrm{l}$ of a BSTFA/pyridine (1:1) mixture.

Urinary organic acids were separated on a Girdel (Suresnes, France) model 30 gas chromatograph coupled with a quadrupole mass spectrometer R 10-10 B (Nermag, Rueil-Malmaison, France) equipped with a PDP 8a computer system (Digital Equipment) with a Sidar data system (Nermag). The chromatographic resolution of organic acids was obtained on a CPSil 5 (25 $\mathrm{m} \times 0.25 \mathrm{~mm}$ ) silica fused capillary column (Chrompack) using helium carrier gas $(2 \mathrm{ml} / \mathrm{mn}$ ) with a Ross injector, the temperature of which was programmed from 110 to $240^{\circ} \mathrm{C}\left(5^{\circ} / \mathrm{min}\right)$. Mass spectrometer conditions were as follows: electron energy, $70 \mathrm{eV}$; source temperature, $180^{\circ} \mathrm{C}$; emission current, $0.2 \mathrm{~mA}$; mass range: 50-550 amu; mass integration: $1 \mathrm{~ms} / \mathrm{amu}$; delay between scan: $0.2 \mathrm{~s}$. The molecular weight of unusual products was determined by chemical ionization with ammonia $(2 \times 10$ 4 Torr). The quantification of organic acids was obtained by measurement of the $[\mathrm{M}-15]$ ions.

\section{RESULTS}

Figure 1 shows the profile of organic acids excreted in the urines of a patient with ZS, patients with NALD presenting a similar pattern. The most prominent characteristic is the presence of DA of various chain length ranging from adipic (C6) to hexadecanedioic (C16) acids. More precisely, three groups of compounds were observed: 1) even-numbered DA with medium (adipic, suberic and sebacic acids) and long-chain (hexadecanedioic acid); 2) odd-numbered DA with medium (pimelic and azelaic acids) and long-chain (undecanedioic, tridecanedioic and pentadecanedioic acids); and 3) medium-chain hydroxy-DA (2and 3-hydroxy-sebacic acids). Several DA were accompanied by the corresponding unsaturated analogue. Quantitation of most of these products is presented in Table 2.

We identified compound 29 in Figure 1 as 2-hydroxy-sebacic acid since it has the same retention time (methylene unit: 20.30) and mass spectrum (Fig. 2) as the pure compound obtained by synthesis.

Products 11, 19, and 26 in Figure 1 were thought to represent unsaturated DA with 7,9 , and 11 carbon atoms, respectively. In the absence of available pure compounds, this was based on the analysis of their mass spectra (Fig. 3) and on the comparison of the retention times and molecular weights of these unsaturated DA with those of the corresponding saturated homologues. The ratio of the $[M-15]$ ion of each unsaturated product to its saturated homologue was in a range of $0.25-1$, an abnormally high value indicating an excess of the unsaturated forms.

Product 31 of Figure 1 was identified as tridecanedioic acid, since its retention time and mass spectrum (Fig. $4 A$ ) were iden-

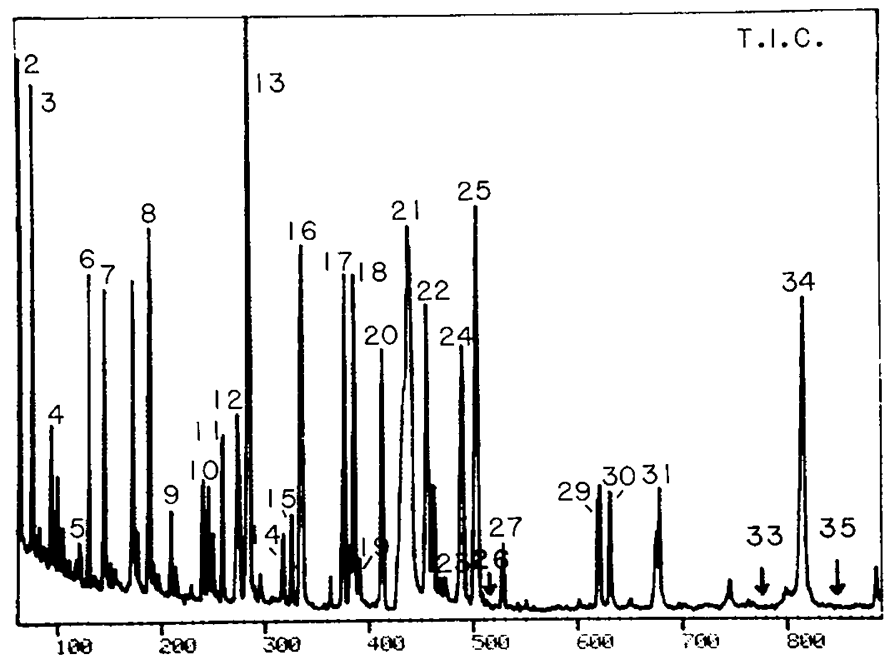

Fig. 1. Urinary organic acid profile of case 1. The peaks are identified as the TMS derivatives of the following acids: 1, 3-hydroxy-butyric; 2, 2hydroxy-isocaproic; 3, succinic; 4, fumaric; 5, glutaric; 6, 2-methylglutaric (internal standard); 7, $n$-butylmalonic (internal standard); 8 , adipic; 9, 3-methyladipic; 10, 2-hydroxy-glutaric; 11, unsaturated pimelic; 12, pimelic; 13, 4-hydroxy-phenylacetic; 14 and 15, unsaturated suberic; 16, suberic; 17, phenylsuccinic (internal standard); 18, homovanillic and aconitic; 19, unsaturated azelaic; 20, azelaic; 21, hippuric; 22, citric; 23 , vanylmandelic and unsaturated sebacic; 24 , sebacic; 25, 4-hydroxyphenyllactic; 26 , unsaturated undecanedioic $\left({ }^{*}\right) ; 27$, undecanedioic; 28 , 4-hydroxy-phenylpyruvic; 29, 2-hydroxy-sebacic; 30, 3-hydroxy-sebacic; 31, tridecanedioic; 32, hydroxy-hippuric; 33, 3-hydroxy-dodecanedioic $\left({ }^{*}\right) ; 34$, pentadecanedioic; 35 , hexadecanedioic $\left({ }^{*}\right)$. The column used was $25 \mathrm{~m} \times 0.2 \mathrm{~mm}$ silica fused capillary column with CPSil 5 and temperature programed at $5^{\circ} / \mathrm{min}$ from $110^{\circ} \mathrm{C}$. $T I C$, total ion current versus scan number;*, product not found in this patient, but present in other cases (retention time indicated by an arrow).

tical to that of the pure compound. Compound 34 was tentatively identified as pentadecanedioic acid on the basis of the relative retention time of this compound compared to that of added tetra- and hexadecanedioic acids, the analysis of its mass spectrum (Fig. $4 B$ ), and its molecular weight [416].

\section{DISCUSSION}

Our patients with ZS and NALD excrete even-numbered free medium-chain DA (adipic, suberic, and sebacic acids), the two latter compounds being more abundant than adipic acid in $6 / 7$ 
Table 2. Levels of urinary DA in patients with ZS and NALD (mmol/mol of creatinine)

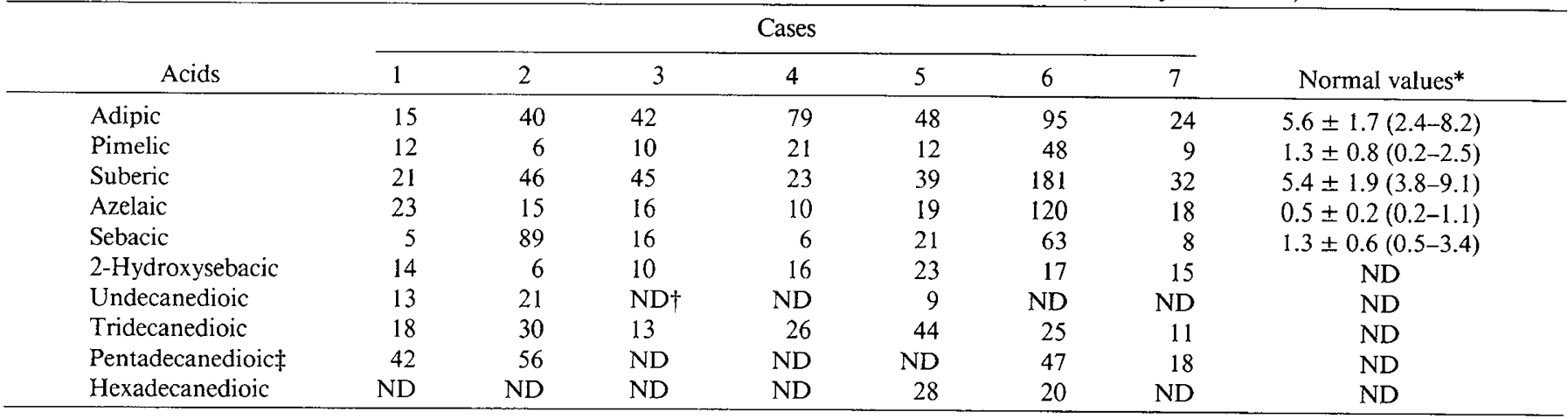

* Mean $\pm \mathrm{SD}$ (range) measured in urines of seven age-matched children fasted for $12 \mathrm{~h}$.

$\uparrow$ Not detectable.

$\ddagger$ Quantitation of pentadecanedioic acid was made using response coefficient of tetradecanedioic acid.

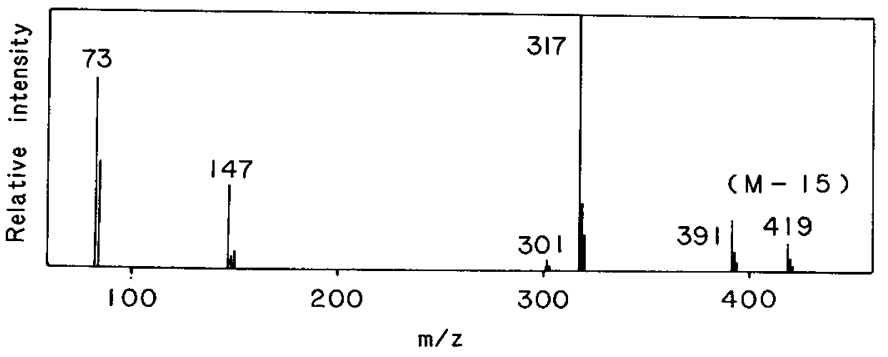

Fig. 2. Electron impact mass spectrum of tri-TMS 2-hydroxy-sebacic acid.
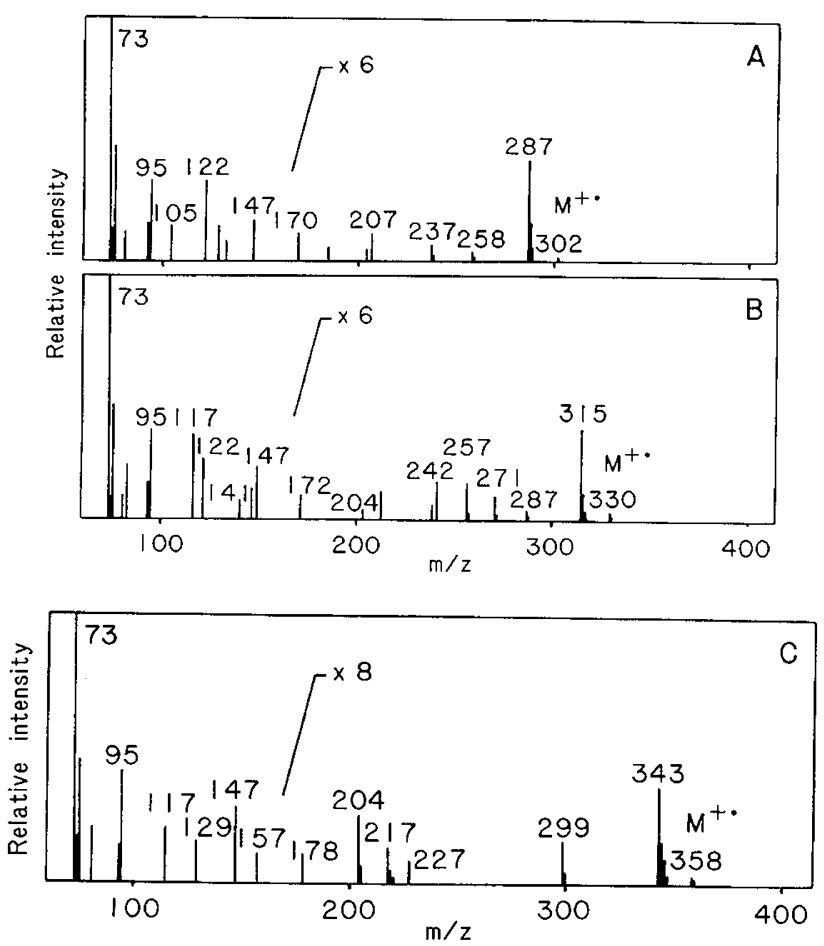

Fig. 3. Electron impact mass spectra of TMS derivatives of the compounds thought to represent unsaturated DA. $A$, heptenedioic; $B$, nonenedioic; $C$, undecenedioic.

cases (Table 2), an observation consistent with that of Björkhem et al. (13) in two patients with ZS. The excretion of DA may result from the increased ketogenic activity of a liver supplied with relatively large amounts of FFA (14), or from an impairment of $\beta$-oxidation of FA in mitochondria and/or peroxisomes (15). Carnitine deficiency, which prevents LCFA to be transported

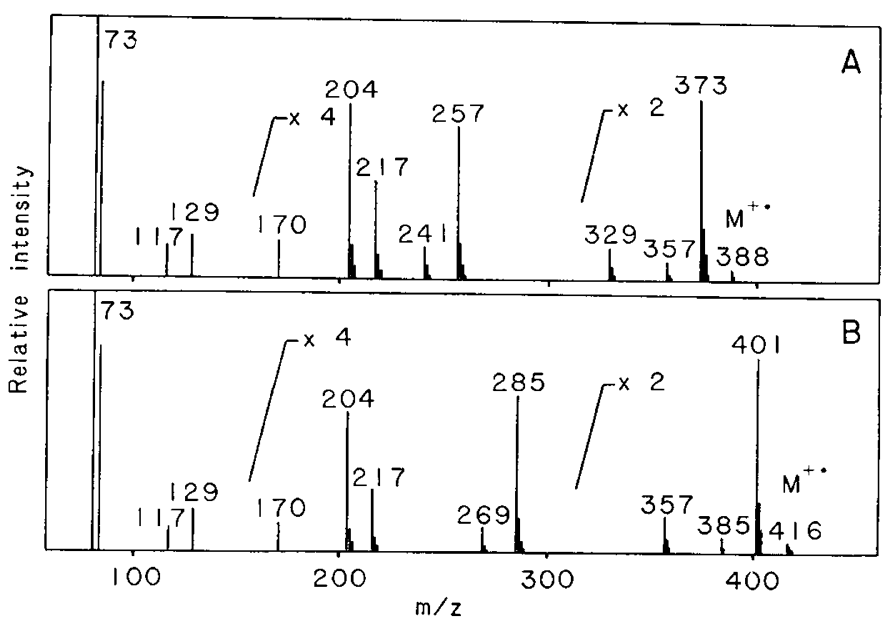

Fig. 4. Electron impact mass spectra representing the TMS derivatives of tridecanedioic acid $(A)$ and of the compound tentatively identified as pentadecanedioic acid $(B)$.

inside mitochondria, thus a well-established cause of dicarboxylic aciduria (16), was excluded since the concentrations of carnitine in the plasma and urine of the patients were normal (table 1).

The most prominent finding in the present study is the accumulation of odd-numbered DA including medium-chain (pimelic and azelaic) as well as long-chain (tri- and pentadecanedioic) acids. The excess of tri- and pentadecanedioic acids can result from two mechanisms (Fig. 5): 1) an increased synthesis of 2-hydroxy-VLCFA leading to odd-numbered VLCFA and 2) a decreased peroxisomal $\beta$-oxidation of odd-numbered VLCFA leading to an accumulation of $\omega$-oxidation products which are uncompletely $\beta$-oxidized.

2-Hydroxy-VLCFA originate mainly from brain, which contains large quantities of VLCFA (C22-C26) as components of myelin cerebrosides and sulfatides (17). These VLCFA undergo $\alpha$-oxidation, i.e. hydroxylation on the $\alpha$-carbon, in microsomes (18-22), then oxidative decarboxylation, giving a FA containing one fewer carbon atom. The synthesis of hydroxy-FA increases rapidly during the myelination period (23), the hydroxylation system being induced during brain development by an increased production of non-hydroxy-VLCFA (20). The accumulation of VLCFA in brain, adrenal glands, and plasma in patients with ZS and NALD (8) is thus likely to induce increased levels of 2hydroxy-analogues. Indirect evidence of the increase of 2-hydroxy-VLCFA in the tissues is the accumulation of odd-numbered-VLCFA (C23:0 and C25:0) in the plasma of these patients (7). Since there is a strong evidence that the peroxisomal catabolism of VLCFA is impaired in ZS and NALD (1-3), oddnumbered VLCFA may rather be directed toward microsomal 


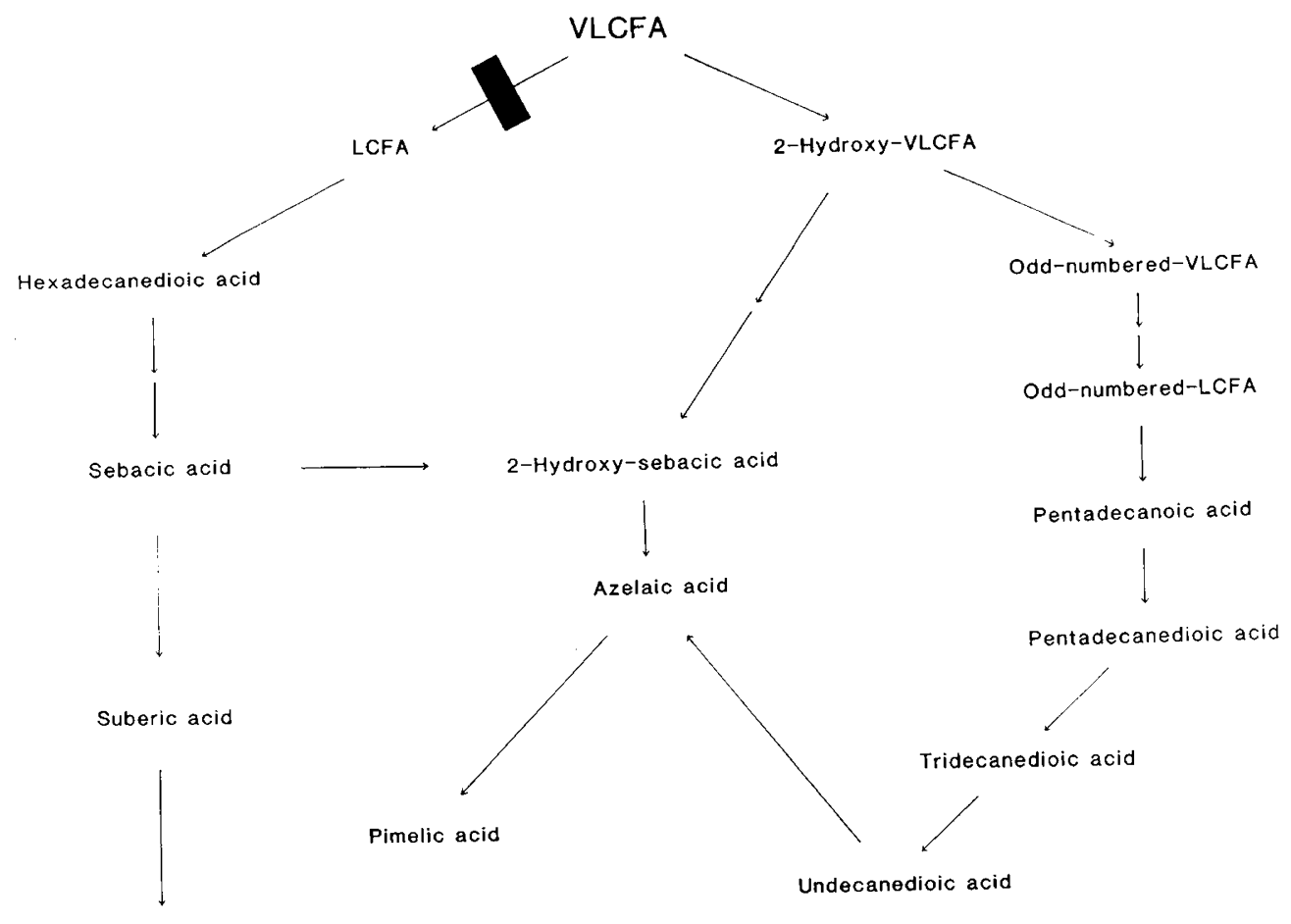

Adipic acid

Fig. 5. Proposed pathway of VLCFA and saturated DA catabolism in ZS and NALD.

$\omega$-oxidation and lead to odd-numbered DA. These odd-numbered DA are then likely to accumulate if their $\beta$-oxidation in mitochondria and/or peroxisomes is impaired (Fig. 5).

Whereas 3-hydroxy-sebacic acid has been observed in defects of $\beta$-oxidation of medium-chain fatty acyl-CoA $(12,24)$ and in ketoacidosis (25), the accumulation of 2-hydroxy-sebacic acid has not been reported in the urine of patients with inborn errors of metabolism. It may arise: 1) from the 2-hydroxylation and $\omega$ oxidation of LCFA and VLCFA and/or 2) from sebacic acid by analogy with the formation of 2-hydroxy-phytanic acid from phytanic acid in the liver (26). The 2-hydroxy-sebacic acid may further be degraded by oxidative decarboxylation, leading to azelaic and pimelic acids (Fig. 5).

The excess of monounsaturated DA may be related to the accumulation of monounsaturated VLCFA, specially C26:1 which is largely increased in ZS and NALD (9).

In conclusion, the accumulation of odd-numbered DA and 2 hydroxy-sebacic acid in ZS and NALD, together with increased plasma levels of VLCFA (5-8), phytanic acid (9), and pipecolic acid (27), as well as the impairment of bile acids (28) and plasmalogens (29-31) synthesis, may be an additional useful markers in this group of peroxisomal disorders.

Aknowledgments. The authors are indebted to Dr. Kamerling who has provided 2-hydroxy-sebacic acid and to Dr. J LalauKeraly who performed carnitine quantitations.

\section{REFERENCES}

1. Kawamura N, Moser HW, Kishimoto X 1981 Very long chain fatty acid oxidation in rat liver. Biochem Biophys Res Commun 99:1216-1225

2. Lazarow PB 1978 Rat liver peroxisomes catalyze the $\beta$ oxidation of fatty acids. J Biol Chem 253:1522-1528

3. Singh I, Moser AE, Goldfischer S, Moser HW 1984 Lignoceric acid is oxidized in the peroxisome: implications for the Zellweger cerebro-hepato-renal syndrome and adrenoleukodystrophy. Proc Natl Acad Sci USA 81:4203-4207

4. Mortensen PB, Kølvraa S, Gregersen N, Rasmussen K 1982 Cyanide-insensitive and clofibrate enhanced $\beta$-oxidation of dodecanedioic acid in rat liver. $\mathrm{An}$ indication of peroxisomal $\beta$-oxidation of $\mathrm{n}$-dicarboxylic acids. Biochim Biophys Acta 713:393-397

5. Aubourg P, Bougnères PF, Rocchiccioli F 1985 Capillary gas chromatographic mass spectrometric measurement of very long chain (C22 to $\mathrm{C} 26)$ fatty acids in microliter plasma samples. J Lipid Res 26:263-267

6. Bakkeren JAJM, Monnens LAH, Trijbels JMF, Maas JM 1984 Serum very long chain fatty acid pattern in Zellweger syndrome. Clin Chim Acta 138:325-331

7. Moser AE, Singh I, Brown III FR, Solish GI, Kelley RI, Benke PJ, Moser HW 1984 The cerebrohepatorenal (Zellweger) syndrome. Increased levels and impaired degradation of very-long-chain fatty acids and their use in prenatal diagnosis. N Engl J Med 310:1141-1146

8. Moser HW, Moser AE, Singh I, O'Neill BP 1984 Adrenoleukodystrophy. Survey of 303 cases: biochemistry, diagnosis and therapy. Ann Neurol 16:628-641

9. Aubourg P, Robain O, Rocchiccioli F, Dancea S, Scotto J 1985 The cerebrohepato-renal (Zellweger) syndrome: lamellar lipid profiles in adrenocortical, hepatic mesenchymal, astrocyte cells and increased levels of very long chain fatty acids and phytanic acid in the plasma. J Neurol Sci 69:9-25

10. Aubourg P, Scotto J, Rocchiccioli F, Feldmann-Pautrat D, Robain O 1985 Neonatal adrenoleukodystrophy. J Neurol Neurosurg Psychiatr (in press)

11. Lalau-Keraly J, Bougnères PF 1984 Apports alimentaires, concentrations circulantes et excrètion de la carnitine en fonction de l'âge chez l'enfant normal. Arch Fr Pediatr 41:715-719

12. Rocchiccioli F, Cartier PH, Bougnères PF 1984 Mass spectrometric identification of abnormal aromatic compounds in the urine of a child with Reye's like syndrome. Biomed Mass Spectrom 11:127-131

13. Björkhem I, Blomstrand S, Haga P, Kase BF, Palonek E, Pedersen JI, Strandvik B, Wikstrom SA 1984 Urinary excretion of dicarboxylic acids from patients with the Zellweger syndrome. Importance of peroxisomes in $\beta$-oxidation of dicarboxylic acids. Biochim Biophys Acta 795:15-19

14. Pettersen JE, Jellum E, Eldjarn L 1971 Short-chain dicarboxylic acids in human urine accompanying ketosis. Scand J Clin Invest 27(suppl 118):69

15. Duran M, De Klerk JBC, Wadman SK, Bruinvis L, Ketting D 1984 The differential diagnosis of dicarboxylic aciduria. J Inher Metab Dis 7(suppl 1):48-51

16. Engel AG, Rebouche CJ 1984 Carnitine metabolism and inborn errors. J Inherited Metab Dis 7(suppl 1):38-43

17. Kishimoto Y, Radin NS 1966 Metabolism of brain glycolipid fatty acids. Lipids 1:47-61

18. Levis GM, Mead JF 1964 An alpha-hydroxy acid decarboxylase in brain microsomes. J Biol Chem 239:77-80

19. Mead JF, Levis GM 1963 Enzymatic decarboxylation of the alpha-hydroxy acids by brain microsomes. Biochem Biophys Res Commun 11:319-324

20. Singh I Kishimoto Y 1979 2-Hydroxylation of lignoceric acid in brain. Subcellular localization of 2-hydroxylation and the requirement for heatstable and heat-labile factors and sphingosine. J Biol Chem 254:7698-7704

21. Hoshi M, Kishimoto Y 1973 Synthesis of cerebronic acid from lignoceric acid by rat brain preparation. J Biol Chem 248:4123-4130

22. Bowen DM, Radin NS 1968 Hydroxy fatty acid metabolism in brain. Adv Lipid Res 6:255-272

23. Murad S, Kishimoto Y 1975 2-Hydroxylation of lignoceric acid to cerebronic acid during brain development. J Biol Chem 250:5841-5846 
24. Bougnères PF, Rocchiccioli F, Kфlvraa S, Hadchouel M, Lalau-Keraly J, Chaussain JL, Wadman SK, Gregersen N 1985 Medium-chain acyl-CoA dehydrogenase deficiency in two siblings with a Reye-like syndrome. $J$ Pediatr 106:918-921

25. Greter J, Lindstedt S, Seeman H, Steen G 1980 3-Hydroxydecanedioic acid and related homologues: urinary metabolites in ketoacidosis. Clin Chem 26:261-265

26. Herndon JH Jr, Steinberg D, Uhlendorf BW, Fales HM 1969 Refsum's disease: characterization of the enzyme defect in cell culture. J Clin Invest 48:10171032

27. Danks DM, Tippett P, Adams C, Campbell P 1975 Cerebro-hepato-renal syndrome of Zellweger. A report of eight cases with comments upon the incidence, the liver lesion, and a fault in pipecolic acid metabolism. J Pediatr $86: 382-387$
28. Hanson RF, Szcepanik-van Leeuwen P, William GC, Grabowski G, Sharp HL 1979 Defects of bile acid synthesis in Zellweger's syndrome. Science 203:1107-1108

29. Datta NS, Wilson GN, Hajra AK 1984 Deficiency of enzymes catalyzing the biosynthesis of glycerol-ether lipids in Zellweger syndrome. $\mathrm{N}$ Engl $\mathrm{J} \mathrm{Med}$ 311:1080-1083

30. Heymans HSA, Bosch Hvd, Schutgens RBH, Tegelaers WHH, Walther JU, Muller-Hocker J, Borst P 1984 Deficiency of plasmalogens in the cerebrohepato-renal (Zellweger) syndrome. Eur J Pediatr 142:10-15

31. Schutgens RBH, Romeyn GJ, Wanders RJA, van den Bosch H, Schrakamp G, Heymans HSA 1984 Deficiency of acyl-CoA:dihydroxyacetone phosphate acyltransferase in patients with Zellweger (cerebro-hepato-renal) syndrome. Biochem Biophys Res Commun 120:179-184 